\title{
Investigación de Mercado de una Granja de Producción de Huevo Libre de Jaula Establecida en Delicias, Chihuahua
}

Market research for a farm wich produce egg free a jail in Delicias, Chihuahua

\section{Magaña Magaña José Eduardo ${ }^{1 凶}$, López Rodríguez Jacqueline ${ }^{2}$, Kiessling Davison Christian Mauricio $^{3}$, Escalera Ochoa Laura ${ }^{4}$, Barrera Torres Julio Cesar ${ }^{5}$}

Profesor de la Facultad de Ciencias Agrícolas y Forestales de la Universidad Autónoma de Chihuahua, ${ }^{1,3,4}$ Profesor de la Facultad de Contaduría y Administración de la Universidad Autónoma de Chihuahua

${ }^{5}$, Estudiante de Maestría en Agronegocios ${ }^{2}$,

凶Autor para correspondencia emagana@uach.mx

Recibido: 29/09/2019

Aceptado: 19/11/2019

\section{RESUMEN}

En la actualidad, el mercado orgánico de alimentos es uno de los más dinámicos ya que los consumidores se informan de los beneficios que tienen al consumirlos. Se analizó tanto la oferta y la demanda existente de huevo al pastoreo libre en la región de Delicias, Chihuahua. El tamaño de la muestra fue de 108 hogares, fueron entrevistados a través de cuestionarios estructurados y aplicados por sector del municipio. El 95\% contestaron que en su hogar se consume huevo. El 84.6\% de los cuales, compraron a la semana presentaciones de $1 \mathrm{~kg}$. y el $15.4 \%$ de $2 \mathrm{~kg}$. Las variables para segmentar el mercado meta fueron el precio y el ingreso familiar mensual. El $13.7 \%$ de los consumidores de huevo pagaron precios de más de 51 pesos y sus ingresos familiares fueron mayores de 8000 pesos al mes. La demanda potencial fue de $337434 \mathrm{~kg}$. La empresa actualmente tiene 23000 gallinas leghorn y las granjas familiares en promedio tienen 200 por lo que si tienen la oferta suficiente para atender el mercado. Se le sugirió a la empresa Distribución cada $3^{\circ}$ día en comercios establecidos y franquicias departamentales, publicidad en radio, redes sociales y folletos de los centros comerciales.

Palabras clave: oferta, demanda, estudio de mercado, avicultura, ecológico.

\begin{abstract}
At present, the organic food market is one of the most dynamic since consumers inform themselves of the benefits which have to consume them. Both was analyzed the supply and the demand of egg to free grazing in the region of Delicias, Chihuahua. The sample size was 108 households which were interviewed through questionnaires, structured and applied by sector of the municipality. 95\% answered that their home consumed egg. The $84.6 \%$ of whom, bought a week presentations of $1 \mathrm{~kg}$. and the $15.4 \%$ of $2 \mathrm{~kg}$. Variables for market segmentation target were the price and the monthly family income. $13.7 \%$ of egg consumers paid prices of more than 51 pesos and their family incomes were
\end{abstract}


greater than 8000 pesos a month. The potential demand was $337434 \mathrm{~kg}$. The company currently has 23000 leghorn hens and family farms on average have 200 so if they have enough supply for meet the market. The distribution company is suggested every 3rd day at established shops and departmental franchises, advertising in radio, social networks and brochures of the malls.

Keywords: supply, demand, market, poultry and ecological research.

\section{.INTRODUCCIÓN}

Actualmente la producción orgánica de alimentos presenta un crecimiento en el mercado nacional y un sólido posicionamiento en la industria alimenticia, debido a las ventajas que los productos brindan a los consumidores y a la responsable conservación del medio ambiente que se genera al producir alimentos sin químicos. En lo que respecta a la producción de huevos orgánicos se debe procurar tener la máxima calidad que se necesita en cuanto a: cuidado y protección del ambiente, seguridad, manejo de suelos, salud y bienestar para las personas. Aun cuando se sabe que existe una gran preocupación de la sociedad por consumir productos sanos, se tiene poca información de los canales de distribución, la demanda actual y potencial de productos orgánicos, particularmente en el estado de Chihuahua (Ávila Rivera, 2015).

La FAO describe la agricultura orgánica o ecológica como un sistema de producción en el cual el principal objetivo es utilizar al máximo los bienes de la tierra, brindando especial cuidado a la fertilidad del suelo y la acción biológica, y por otro lado reduciendo el empleo de los recursos no renovables, sin el uso de fertilizantes y plaguicidas sintéticos defendiendo así el medio ambiente y la salud humana (FAO, 2018).

Actualmente esta producción representa ingresos anuales de 400 millones de dólares, siendo el café, miel, cacao, aguacate, mango, piña y plátano los más cotizados en el mercado
(Aguilar, 2015). Con lo que respecta al ramo avícola, lo ecológico también está creando tendencias positivas entre los consumidores, especialmente en los que están dispuestos a adquirirlos con un incremento del 30\%. Los indicadores oficiales disponibles de Senasica mencionan que, en 2014, los pollos en engorda bajo la certificación de la Ley de Productos Orgánicos sumaban 266 mil aves, cifra que subió a 354 mil en 2015. En tanto el número de gallinas ponedoras en 2014 era de 3 mil, las cuales pasaron a 46 mil en 2015 (Valadez Delgado, 2018).

\section{Antecedentes}

- Nombre de la empresa: 18 Hermanos, S.P.R. de R.L.

- Número de socios: 2.

- Fecha de inicio de operaciones: año 2014.

- Tamaño de la parvada: 23000 gallinas Leghorn

- Capacidad de producción diaria: 19550 unidades.

- Mercado de competencia: Ciudades de Chihuahua y Delicias.

- Canal de distribución: Centros comerciales y al público como minorista.

La empresa avícola es un negocio familiar con historia generacional, en donde se inicia con la enseñanza del padre al establecer una engorda de pollo en el jardín de su casa. Este negocio empezó con la inquietud de tener una mejor calidad en la dieta alimenticia para todos los 
integrantes de la familia, desde los más chiquillos hasta los no tan pequeños. La producción comenzó siendo de consumo propio $\mathrm{y}$ sin fines de lucro, luego esa necesidad comenzó a crecer y fue por el año 2015, con aproximadamente 40 aves, que la empresa comenzó a especializarse en la producción del huevo a través del sistema conocido como pastoreo rotacional en el cual la pradera se divide en potreros iguales alrededor del gallinero de esta manera se le permite a las aves que siempre coman alfalfa fresca, fertilicen la tierra y le dé oportunidad a la pradera de descansar y así se pueda garantizar siempre la calidad, el sabor y la salud, para eficientar su producto $\mathrm{y}$ ofrecerlo a precios razonables $\mathrm{y}$ accesibles para todos.

\section{Planteamiento del problema de la investigación}

No se cuenta con información de mercado que contribuya a realizar la toma de decisiones correcta con respecto a las estrategias de comercialización del producto en el mercado local.

Es necesario conocer y establecer el mercado objetivo para que de esta manera la empresa se desarrolle de manera competitiva construyendo canales de distribución en la región.

\section{Justificación de la investigación}

Es indispensable que la empresa tenga información suficiente y útil de mercado del huevo de origen saludable, ya que así sabrá con exactitud el perfil de consumo que conduzca a determinar el mercado potencial.

\section{Revisión de literatura}

La avicultura dedicada a la producción de huevo sano se le conoce como producción de huevo de libre pastoreo. Que es un proceso en el que las gallinas pasan la mayor parte de su vida fuera de su gallinero portátil, el cual solamente lo utilizan para protegerse, dormir y poner sus huevos (Garcia Hernández, 2018).

La comercialización consiste en planear y organizar las actividades necesarias para posicionar un producto o servicio para que los consumidores lo conozcan y lo consuman (Kotler \& Armstrong, 2008). Esta definición de comercialización nos conduce a conocer el consumo de huevo en general y huevo de libre pastoreo en la región, basado en la oferta y demanda regional.

Por otro lado, Thovar Bermejo (2019), en su artículo denominado "Huevos y Ovoproductos" declara que para determinar correctamente la comercialización del huevo, se tiene que conocer a fondo el sistema de producción del mismo así como los estándares de calidad que apliquen en cada localidad, sugiriendo que en la etiqueta se debe de mencionar la fecha de consumo preferente del huevo, la forma de producción, contenido nutrimental, además de toda la información adicional promocional de la empresa. Al conocer estos datos y cumpliendo con las normas será de una manera más fácil la colocación del producto en el mercado.

Con el estudio realizado por estudiantes de la Universidad de Santo Tomás de Colombia denominado "Plan de negocios de huevo libre de jaula" coinciden en que el consumo de huevo es de 3 a 5 piezas por semana por persona y que el mercado objetivo para huevo libre de jaula son todas aquellas personas que estén dispuestas a balancear y mejorar sus hábitos alimenticios consumiendo productos que no contengan contaminantes que afecten su salud, además que de que tengan un proceso de producción más amigable con el medio ambiente (Borbón Riveros, Chaves Chinome, \& Moreno Cabra, 2017).

La investigación realizada por Uriel Reyes Hernández del Instituto Tecnológico de 
Tlaxiaco, Oaxaca llamada "Diseño de un plan de negocios para una empresa productora $\mathrm{y}$ comercializadora de huevo libre de jaula" menciona que el mercado de productos orgánicos es un mercado que viene en crecimiento, discreto pero viable; aunado a eso que se trata de un producto de la canasta básica y que además es consumido por la mayoría de la población, un producto altamente demandable (Reyes Hernández, 2014).

\section{MATERIALES Y MÉTODOS}

La metodología para la obtención de información del diagnóstico situacional consistió en una investigación de mercado mediante el proceso que propone Malhotra (2008) que consiste en los siguientes pasos:

Definición del problema- En la entrevista con los expertos y dueños de la empresa se identifica que en un futuro no muy lejano, ante la compra de nuevas aves ponedoras de libre pastoreo se espera un producción mayor de huevo, el cual es necesario colocar para su venta.

Desarrollo del enfoque del problema- Por lo que se debe de establecer y desarrollar un plan de comercialización, que incluya mayores líneas de distribución del producto.

Formulación del diseño de investigaciónDeterminar si existe mercado que demande este tipo de producto en la ciudad de Delicias.
Trabajo de campo o recopilación de datos- A través de 108 cuestionarios con preguntas referentes a oferta y demanda aplicados por medio de entrevistas personales con el número determinado en la muestra.

Preparación y análisis de datos- Se utilizará el software IBM SPSS V.22

Preparación y presentación del informe de datos. Se codificará el cuestionario y se creará una base de datos.

Se elaboró un cuestionario formal estructurado con 19 preguntas para la demanda y siete para la oferta.

La población de estudio fueron los núcleos familiares que viven en los diferentes sectores en Delicias, Chihuahua.

El marco muestral considerado fue el listado de los hogares existentes en la ciudad proporcionado por la Presidencia Municipal de Delicias.

La unidad de análisis para la investigación fueron las familias de Delicias, Chihuahua.

El tipo de muestreo fue probabilístico, ya que todos los elementos, unidades de observación, unidades de análisis o unidades de muestreo tuvieron la misma oportunidad de ser seleccionados.

El tamaño de la muestra se obtuvo utilizando el método de intervalo de confianza considerando un $5 \%$ de nivel de significancia, un $95 \%$ de nivel de confianza utilizando un $7 \%$ de la media muestral como error permitido dando una muestra de 108 cuestionarios.

Tabla 1. Participación relativa de las familias por sector.

\begin{tabular}{r|ccc}
\multicolumn{1}{c|}{ Sector } & Número & $\begin{array}{c}\text { Participación } \\
\text { relativa \% }\end{array}$ & Muestra \\
\hline Norte & 4,871 & 9.0 & 11 \\
\hline Sur & 20,600 & 40.0 & 43 \\
\hline Oriente & 17,349 & 34.0 & 37 \\
\hline Poniente & 8,704 & 17.0 & 17 \\
Total & $\mathbf{5 1 , 5 2 4}$ & $\mathbf{1 0 0}$ & $\mathbf{1 0 8}$
\end{tabular}

Fuente: Catastro de ciudad Delicias, Chihuahua. 
La selección de la muestra fue probabilístico aleatorio.

El trabajo de investigación se realizó en ciudad Delicias, Chihuahua entre agosto de 2017 hasta junio de 2019.

\section{Procesamiento y análisis de la información}

Se procesó la información en procesadores de datos numéricos y de texto.

Se analizaron los datos mediante gráficos, figuras, tablas de frecuencia y tablas de contingencias y se utilizó la prueba estadística no paramétrica de $\mathrm{X}^{2}$ como prueba de hipótesis para ver dependencias entre las variables. Para de esta manera analizar la información recabada e interpretarla para concluir con los objetivos planteados en un principio en este documento.

\section{RESULTADOS}

Para efectos de este estudio se verificó la confiabilidad del instrumento a través del coeficiente Alpha de Cronbach que permite estimar la fiabilidad de un instrumento a través de un conjunto de ítems que se esperan midan el mismo constructo o dimensión teórica. Como criterio general, (George \& Mallery, 2003, pág. 231) sugieren las recomendaciones siguientes para evaluar los coeficientes de alfa de Cronbach:

Tabla 2. Nivel de confiabilidad del instrumento.

\begin{tabular}{|c|c|}
\hline Coeficiente alfa & Grado \\
\hline$>0.9$ & Excelente \\
\hline$>0.8$ & Bueno \\
\hline$>0.7$ & Aceptable \\
\hline$>0.6$ & Cuestionable \\
\hline$>0.5$ & Pobre \\
\hline
\end{tabular}

Fuente: (George \& Mallery, 2003, pág. 231)

Tabla 3. Nivel de confiabilidad del instrumento.

\begin{tabular}{|l|l|}
\hline Alfa de Cronbach & $\begin{array}{l}\text { Número de } \\
\text { elementos }\end{array}$ \\
\hline 0.835 & 18 \\
\hline
\end{tabular}

Fuente: Obtenido del análisis de datos de la muestra.

Como se indicó en la tabla 3, el coeficiente de Alfa de Cronbach de las 18 preguntas con escala de Likert, arrojó como resultado 0.835 . De acuerdo a la escala de George y Mallery (2003), el resultado se consideró bueno. Por lo tanto, todos los constructos que se hacen en este estudio de caso son válidos desde el punto de vista conceptual teórico.

Para el estudio de la oferta de huevo en la ciudad se aplicó la encuesta a los centros comerciales de la ciudad reconocidos oficialmente. Al analizar estos datos se muestran que aproximadamente las tiendas compran a sus proveedores 610,000 piezas mensuales, de las cuales 13,728 corresponden a huevo producido libre de jaula lo que representa el $2.25 \%$ de la compra; mencionando las personas encargadas de las tiendas que dicha cantidad se debe a que primeramente se vende el huevo convencional ya que los clientes desconocen que también se venden este tipo de productos en los supermercados y rara vez se tiene promoción del huevo libre de jaula, dicho dato coincide con lo mencionado en la investigación realizada por Mendoza (2018) en la que concluye que es de suma importancia la publicidad que se le dé a estos productos ya que 
aún los beneficios del mercado orgánico se desconocen entre la población.

En la figura 1 se puede identificar que la población encuestada consume en su mayoría el huevo como alimento en su hogar registrándose más de un $95 \%$ de los entrevistados, esto coincide con lo señalado por Valadez (2018) quien menciona que la compra de este alimento se ubica por encima de los $23.7 \mathrm{~kg}$ y que el consumo de huevo en México registra crecimientos constantes atribuyendo a diversos factores como el precio, accesibilidad, calidad, preparación practica y el ingreso per cápita de la población. Esta información puede explicar el alto consumo registrado de este producto.

Tabla 4. Consumo de huevo en el hogar.

\begin{tabular}{|c|c|}
\hline ¿Consumen huevo en su casa? & Porcentaje \\
\hline $\mathrm{Si}$ & 95.05 \\
\hline $\mathrm{No}$ & 4.05 \\
\hline
\end{tabular}

Fuente: Obtenido del análisis de datos de la muestra.

De la población que consume huevo se identificó que el $27 \%$ conocen los beneficios del consumo de huevo de libre pastoreo, mismos que a su vez están dispuestos a adquirirlos si los encontrasen en establecimientos de fácil acceso; estos datos coinciden con la investigación "Plan de negocios de huevo libre de jaula" realizada por estudiantes de la Universidad de Santo Tomás de Bogotá, Colombia, en la que mencionan que la mayoría de sus encuestados prefiere realizar las compras de este producto en supermercados seguido por las tiendas de barrio. Un factor importante para el éxito de los productos de origen natural es su accesibilidad con respecto al consumidor, así lo menciona Gómez et al, (2002), en el que el principal canal de comercialización son los supermercados, seguido por tiendas especializadas y por último ventas directas.

Para analizar las relaciones entre dos variables se utilizó la tabla cruzada o de contingencia para las variables categóricas para la cual se necesita formular hipótesis de trabajo. Una vez establecidas las relaciones, en caso de existir, se tiene que encontrar la suficiente evidencia estadística que permita aceptar o rechazar la hipótesis nula de independencia de variables, para lo cual se utiliza el estadístico Chicuadrada.

Se analizó la correlación que existe entre la variable consumo y la variable precio, en la que se plantearon las siguientes hipótesis:

$\mathrm{H}_{0}=$ el consumo de huevo en casa no está relacionado con el precio del huevo que se paga.

$\mathrm{H}_{\mathrm{a}}=\mathrm{el}$ consumo de huevo en casa está relacionado con el precio del huevo que se paga.

Y de acuerdo a que el valor crítico en tablas (12.5916) es menor que el valor calculado de chi cuadrada se rechaza la hipótesis nula y se tiene la suficiente evidencia estadística que la variable consumo de huevo está relacionado con el precio; siendo así que los respondientes manifestaron consumir huevo en su casa en un $95 \%$, y al cruzar la información con el precio por kilogramo de huevo que compra, se obtuvo que el $21.1 \%$ paga entre $\$ 25$ - $\$ 30$, el $28.4 \%$ paga entre $\$ 31-\$ 35$ y el $27.4 \%$ paga en un rango entre $\$ 36$ - $\$ 40$. El segmento que nos interesa lo representan aquellos consumidores que pagan más de $\$ 51$ en virtud de que el precio de mercado del huevo libre de jaula está entre $\$ 55$ y $\$ 60$ por kilogramo. Este grupo de consumidores está representado por el $13.7 \%$ de 
los respondientes, en otras palabras, siendo un total de 6,852 núcleos familiares.

Teniendo el dato del número de familias que conforman nuestro mercado objetivo 6852, el 13.7\% y sabiendo que 5797 familias en cada compra adquieren un kilogramo de huevo y el resto $2 \mathrm{kgs}$. Y teniendo la información de la frecuencia de compra se obtiene la siguiente tabla, en la cual se determina los kilogramos anuales demandados por el mercado:

Tabla 5. Frecuencia y cantidad de consumo familiar.

\begin{tabular}{|c|c|c|c|}
\hline & $1 \mathrm{~kg}$. & 2 kgs. & Total kgs. anuales \\
\hline Cada 2 días & $\begin{array}{c}348 \text { familias } \\
\text { (63 } 474 \text { kgs. anuales) }\end{array}$ & 0 & 63474 \\
\hline 1 vez por semana & $\begin{array}{c}3912 \text { familias } \\
\text { (172 } 118 \mathrm{kgs} . \text { anuales) }\end{array}$ & $\begin{array}{c}904 \text { familias } \\
\text { (47 } 024 \text { kgs. anuales) }\end{array}$ & 219142 \\
\hline Cada 15 días & $\begin{array}{c}2124 \text { familias } \\
\text { (46722 kgs. anuales) }\end{array}$ & $\begin{array}{c}151 \text { familias } \\
\text { (3 } 923 \text { kgs. anuales) }\end{array}$ & 59645 \\
\hline $1 \mathrm{vez}$ por mes & $\begin{array}{c}348 \text { familias } \\
\text { (4 } 173 \mathrm{kgs} . \text { anuales) }\end{array}$ & 0 & 4173 \\
\hline Total & 5797 familias & 1055 familias & 337434 kgs \\
\hline
\end{tabular}

Fuente: Obtenido del análisis de datos de la muestra.

Al analizar la relación que existe entre las variables ingreso mensual y precio que está dispuesto a pagar por el producto se encontró que el $54.2 \%$ del segmento meta tiene un ingreso entre $\$ 8000$ y $\$ 12000$ al mes. La mayor parte que paga ese precio y está dispuesto a consumirlo tiene un ingreso familiar de más de $\$ 8000$ por mes. La información estableció que el consumo de huevo libre de jaula es de una clase media baja a media alta.

Un factor importante para el éxito de los productos de origen natural es su accesibilidad con respecto al consumidor, así lo menciona
Gómez et al, (2002), en el que el principal canal de comercialización son los supermercados, seguido por tiendas especializadas y por último ventas directas. La distribución fuerte del producto debe de ser en los supermercados de los sectores oriente y poniente de la ciudad, ya que es donde se ubicaron los domicilios de estos consumidores que son mayores de 41 años de edad, este dato coincide con el mismo autor que en su estudio muestra que el $29 \%$ de los consumidores de productos orgánicos se encuentra en los rangos de edad entre 30 y 59 años (Gómez, Gómez, \& Schwentesius, 2002).

Tabla 6. Pruebas de chi-cuadrado de las variables precio y frecuencia de compra.

\begin{tabular}{|l|l|l|l|}
\hline & Valor & gl & Sig. asintótica (2 caras) \\
\hline Chi-cuadrado de Pearson & $97.163^{\mathrm{a}}$ & 24 & .000 \\
Razón de verosimilitud & 51.647 & 24 & .001 \\
Asociación lineal por & 41.423 & 1 & .000 \\
lineal & 100 & & \\
N de casos válidos & & \\
\hline
\end{tabular}

Fuente: Obtenido del análisis de datos de la muestra. $\alpha=0.05, \mathrm{gl}=24$. Valor crítico $=36.4150$. Valor calculado $=97.163$

Con el valor crítico obtenido que es menor que el valor calculado se rechaza la hipótesis nula ya que el resultado de correlación entre las variables muestra la dependencia entre el precio 
Tabla 7. Relación entre precio y frecuencia de compra.

\begin{tabular}{|c|c|c|c|c|c|c|c|c|c|}
\hline & \multicolumn{7}{|c|}{ Precio que paga actualmente } & \multirow[b]{2}{*}{ Total } \\
\hline & & $\begin{array}{l}\$ 25-\$ \\
30\end{array}$ & $\begin{array}{l}\$ 31 \\
\$ 35\end{array}$ & $\begin{array}{l}\$ 36 \\
\$ 40\end{array}$ & $\begin{array}{l}\$ 41 \\
\$ 45\end{array}$ & $\begin{array}{l}\$ 46 \\
\$ 50\end{array}$ & \begin{tabular}{|ll} 
Más de \\
$\$ 51$
\end{tabular} & $\begin{array}{l}\text { No } \\
\text { consume }\end{array}$ & \\
\hline \multirow[t]{5}{*}{$\begin{array}{l}\text { ¿Con } \\
\text { frecuencia } \\
\text { adquiere? }\end{array}$} & $\begin{array}{l}\text { qué Cada } 2 \text { días } \\
\text { lo }\end{array}$ & $\begin{array}{l}3 \\
42.9 \% \\
15.0 \%\end{array}$ & $\begin{array}{l}3 \\
42.9 \% \\
11.1 \% \\
\end{array}$ & $\begin{array}{l}0 \\
0.0 \% \\
0.0 \%\end{array}$ & $\begin{array}{l}0 \\
0.0 \% \\
0.0 \%\end{array}$ & $\begin{array}{l}0 \\
0.0 \% \\
0.0 \%\end{array}$ & $\begin{array}{l}1 \\
14.3 \% \\
7.7 \% \\
\end{array}$ & $\begin{array}{l}0 \\
0.0 \% \\
0.0 \%\end{array}$ & $\begin{array}{l}7 \\
100.0 \% \\
7.0 \% \\
\end{array}$ \\
\hline & $\begin{array}{l}\text { Una vez } \\
\text { por semana }\end{array}$ & $\begin{array}{l}11 \\
19.3 \% \\
55.0 \%\end{array}$ & $\begin{array}{l}17 \\
29.8 \% \\
63.0 \% \\
\end{array}$ & $\begin{array}{l}18 \\
31.6 \% \\
69.2 \% \\
\end{array}$ & $\begin{array}{l}2 \\
3.5 \% \\
33.3 \% \\
\end{array}$ & $\begin{array}{l}2 \\
3.5 \% \\
66.7 \% \\
\end{array}$ & $\begin{array}{l}7 \\
12.3 \% \\
53.8 \% \\
\end{array}$ & $\begin{array}{l}0 \\
0.0 \% \\
0.0 \%\end{array}$ & \begin{tabular}{|l|}
57 \\
$100.0 \%$ \\
$57.0 \%$ \\
\end{tabular} \\
\hline & $\begin{array}{l}\text { Cada } \\
\text { días }\end{array}$ & $\begin{array}{l}6 \\
22.2 \% \\
30.0 \% \\
\end{array}$ & \begin{tabular}{|l|}
5 \\
$18.5 \%$ \\
$18.5 \%$ \\
\end{tabular} & $\begin{array}{l}6 \\
22.2 \% \\
23.1 \% \\
\end{array}$ & \begin{tabular}{|l|}
4 \\
$14.8 \%$ \\
$66.7 \%$ \\
\end{tabular} & $\begin{array}{l}1 \\
3.7 \% \\
33.3 \% \\
\end{array}$ & \begin{tabular}{|l|}
5 \\
$18.5 \%$ \\
$38.5 \%$ \\
\end{tabular} & $\begin{array}{l}0 \\
0.0 \% \\
0.0 \% \\
\end{array}$ & \begin{tabular}{|l|}
27 \\
$100.0 \%$ \\
$27.0 \%$ \\
\end{tabular} \\
\hline & $\begin{array}{l}\text { Una vez al } \\
\text { mes }\end{array}$ & $\begin{array}{l}0 \\
0.0 \% \\
0.0 \% \\
\end{array}$ & $\begin{array}{l} \\
40.0 \% \\
7.4 \% \\
\end{array}$ & $\begin{array}{l}2 \\
40.0 \% \\
7.7 \% \\
\end{array}$ & $\begin{array}{l}0 \\
0.0 \% \\
0.0 \% \\
\end{array}$ & $\begin{array}{l}0 \\
0.0 \% \\
0.0 \% \\
\end{array}$ & $\begin{array}{l}0 \\
0.0 \% \\
0.0 \% \\
\end{array}$ & $\begin{array}{l}1 \\
20.0 \% \\
20.0 \% \\
\end{array}$ & \begin{tabular}{|l|}
5 \\
$100.0 \%$ \\
$5.0 \%$ \\
\end{tabular} \\
\hline & $\begin{array}{l}\text { No } \\
\text { consume }\end{array}$ & $\begin{array}{l}0 \\
0.0 \% \\
0.0 \% \\
\end{array}$ & $\begin{array}{l}0 \\
0.0 \% \\
0.0 \% \\
\end{array}$ & $\begin{array}{l}0 \\
0.0 \% \\
0.0 \% \\
\end{array}$ & $\begin{array}{l}0 \\
0.0 \% \\
0.0 \% \\
\end{array}$ & $\begin{array}{l}0 \\
0.0 \% \\
0.0 \% \\
\end{array}$ & $\begin{array}{l}0 \\
0.0 \% \\
0.0 \% \\
\end{array}$ & $\begin{array}{l}4 \\
100.0 \% \\
80.0 \% \\
\end{array}$ & \begin{tabular}{|l|}
4 \\
$100.0 \%$ \\
$4.0 \%$ \\
\end{tabular} \\
\hline \multicolumn{2}{|l|}{ Total } & $\begin{array}{l}20 \\
20.0 \% \\
100.0 \%\end{array}$ & $\begin{array}{l}27 \\
27.0 \% \\
100.0 \%\end{array}$ & $\begin{array}{l}26 \\
26.0 \% \\
100.0 \%\end{array}$ & $\begin{array}{l}6 \\
6.0 \% \\
100.0 \%\end{array}$ & $\begin{array}{l}3 \\
3.0 \% \\
100.0 \%\end{array}$ & $\begin{array}{l}13 \\
13.0 \% \\
100.0 \%\end{array}$ & $\begin{array}{l}5 \\
5.0 \% \\
100.0 \%\end{array}$ & \begin{tabular}{|l|}
100 \\
$100.0 \%$ \\
$100.0 \%$
\end{tabular} \\
\hline
\end{tabular}

Fuente: Obtenido del análisis de datos de la muestra.

Del segmento que paga actualmente el precio de huevo libre de jaula, se observó la mayor parte que la frecuencia de compra es de 1 vez por semana $(12.3 \%)$ y cada 15 días $(18.5 \%)$.

$\mathrm{H}_{0}=$ La variable del ingreso mensual es independiente de la variable precio que estaría dispuesto a pagar.

$\mathrm{H}_{\mathrm{a}}=\mathrm{La}$ variable del ingreso mensual es dependiente de la variable precio estaría dispuesto a pagar.

Tabla 8. Pruebas de chi-cuadrado de la variable ingreso mensual y precio que estaría dispuesto a pagar.

\begin{tabular}{|l|l|l|l|}
\hline & Valor & Gl & Sig. asintótica (2 caras) \\
\hline Chi-cuadrado de Pearson & $61.516^{\mathrm{a}}$ & 42 & .026 \\
Razón de verosimilitud & 43.307 & 42 & .415 \\
Asociación lineal por & 25.639 & 1 & .000 \\
lineal & 99 & & \\
N de casos válidos & & \\
\hline
\end{tabular}

Fuente: Obtenido del análisis de datos de la muestra.

$\alpha=0.05, \mathrm{gl}=42$

Valor crítico $=55.7585$

Valor calculado $=61.516$

Con el valor crítico obtenido que es menor que el valor calculado se rechaza la hipótesis nula ya que el resultado de correlación entre las variables muestra la dependencia entre la percepción del ingreso mensual y el precio que estaría dispuesto a pagar. 
Tabla 9. Relación entre ingreso mensual y precio que estaría dispuesto a pagar.

\begin{tabular}{|c|c|c|c|c|c|c|c|c|c|}
\hline & \multicolumn{7}{|c|}{ Nivel de ingreso familiar mensual } & \multirow[b]{2}{*}{ Total } \\
\hline & & \begin{tabular}{|lr}
\multicolumn{2}{|l}{ Menos } \\
de & $\$ 3$ \\
000 & al \\
mes &
\end{tabular} & $\begin{array}{l}\text { Entre } \\
\$ 3001 \quad y \\
\$ 5000\end{array}$ & $\begin{array}{l}\text { Entre } \\
\$ 5001 \text { y } \\
\$ 8000\end{array}$ & $\begin{array}{l}\text { Entre } \\
\$ 8001 \text { y } \\
\$ 12000\end{array}$ & \begin{tabular}{|l} 
Entre \\
$\$ 12001$ \\
$\mathrm{y}$ \\
$\$ 15000$
\end{tabular} & $\begin{array}{l}\text { Más de } \\
\$ 15001 \\
\text { al mes }\end{array}$ & $\begin{array}{l}\text { No } \\
\text { consume }\end{array}$ & \\
\hline \multirow{8}{*}{$\begin{array}{l}\text { ¿Cuánto estaría } \\
\text { dispuesto } \\
\text { pagar por un } \\
\text { kilogramo de } \\
\text { huevo } \\
\text { orgánico? }\end{array}$} & $\begin{array}{l}\text { Entre } \\
\$ 40 \\
\$ 50\end{array}$ & $\begin{array}{l}2 \\
6.3 \% \\
66.7 \%\end{array}$ & $\begin{array}{l}8 \\
25.0 \% \\
25.8 \%\end{array}$ & $\begin{array}{l}10 \\
31.3 \% \\
41.7 \%\end{array}$ & $\begin{array}{l}6 \\
18.8 \% \\
25.0 \%\end{array}$ & $\begin{array}{l}6 \\
18.8 \% \\
60.0 \%\end{array}$ & $\begin{array}{l}0 \\
0.0 \% \\
0.0 \%\end{array}$ & $\begin{array}{l}0 \\
0.0 \% \\
0.0 \%\end{array}$ & $\begin{array}{l}32 \\
100.0 \% \\
32.3 \%\end{array}$ \\
\hline & $\begin{array}{l}\text { Entre } \\
\$ 51 \\
\$ 60\end{array}$ & $\begin{array}{l}1 \\
3.7 \% \\
33.3 \% \\
\end{array}$ & $\begin{array}{l}7 \\
25.9 \% \\
22.6 \% \\
\end{array}$ & $\begin{array}{l}5 \\
18.5 \% \\
20.8 \% \\
\end{array}$ & \begin{tabular}{|l|}
10 \\
$37.0 \%$ \\
$41.7 \%$ \\
\end{tabular} & $\begin{array}{l}2 \\
7.4 \% \\
20.0 \% \\
\end{array}$ & $\begin{array}{l}2 \\
7.4 \% \\
33.3 \% \\
\end{array}$ & $\begin{array}{l}0 \\
0.0 \% \\
0.0 \% \\
\end{array}$ & $\begin{array}{l}27 \\
100.0 \% \\
27.3 \% \\
\end{array}$ \\
\hline & $\begin{array}{l}\text { Entre } \\
\$ 61 \quad y \\
\$ 70\end{array}$ & $\begin{array}{l}0 \\
0.0 \% \\
0.0 \% \\
\end{array}$ & $\begin{array}{l}0 \\
0.0 \% \\
0.0 \% \\
\end{array}$ & \begin{tabular}{|l|}
2 \\
$33.3 \%$ \\
$8.3 \%$ \\
\end{tabular} & \begin{tabular}{|l|}
2 \\
$33.3 \%$ \\
$8.3 \%$ \\
\end{tabular} & \begin{tabular}{|l|}
1 \\
$16.7 \%$ \\
$10.0 \%$ \\
\end{tabular} & \begin{tabular}{|l|}
1 \\
$16.7 \%$ \\
$16.7 \%$ \\
\end{tabular} & $\begin{array}{l}0 \\
0.0 \% \\
0.0 \% \\
\end{array}$ & $\begin{array}{l}6 \\
100.0 \% \\
6.1 \% \\
\end{array}$ \\
\hline & $\begin{array}{l}\text { Entre } \\
\$ 71 \\
\$ 80\end{array}$ & $\begin{array}{l}0 \\
0.0 \% \\
0.0 \%\end{array}$ & $\begin{array}{l}1 \\
50.0 \% \\
3.2 \% \\
\end{array}$ & $\begin{array}{l}0 \\
0.0 \% \\
0.0 \%\end{array}$ & $\begin{array}{l}1 \\
50.0 \% \\
4.2 \% \\
\end{array}$ & $\begin{array}{l}0 \\
0.0 \% \\
0.0 \% \\
\end{array}$ & $\begin{array}{l}0 \\
0.0 \% \\
0.0 \% \\
\end{array}$ & $\begin{array}{l}0 \\
0.0 \% \\
0.0 \%\end{array}$ & $\begin{array}{l}2 \\
100.0 \% \\
2.0 \%\end{array}$ \\
\hline & $\begin{array}{l}\text { Más de } \\
\$ 80\end{array}$ & $\begin{array}{l}0 \\
0.0 \% \\
0.0 \% \\
\end{array}$ & $\begin{array}{l}2 \\
100.0 \% \\
6.5 \% \\
\end{array}$ & $\begin{array}{l}0 \\
0.0 \% \\
0.0 \% \\
\end{array}$ & $\begin{array}{l}0 \\
0.0 \% \\
0.0 \% \\
\end{array}$ & $\begin{array}{l}0 \\
0.0 \% \\
0.0 \% \\
\end{array}$ & $\begin{array}{l}0 \\
0.0 \% \\
0.0 \% \\
\end{array}$ & $\begin{array}{l}0 \\
0.0 \% \\
0.0 \%\end{array}$ & $\begin{array}{l}2 \\
100.0 \% \\
2.0 \% \\
\end{array}$ \\
\hline & $\begin{array}{l}\text { Lo que } \\
\text { cueste }\end{array}$ & $\begin{array}{l}0 \\
0.0 \% \\
0.0 \%\end{array}$ & $\begin{array}{l}7 \\
46.7 \% \\
22.6 \%\end{array}$ & $\begin{array}{l}5 \\
33.3 \% \\
20.8 \%\end{array}$ & $\begin{array}{l}2 \\
13.3 \% \\
8.3 \% \\
\end{array}$ & $\begin{array}{l}0 \\
0.0 \% \\
0.0 \%\end{array}$ & $\begin{array}{l}1 \\
6.7 \% \\
16.7 \% \\
\end{array}$ & $\begin{array}{l}0 \\
0.0 \% \\
0.0 \%\end{array}$ & $\begin{array}{l}15 \\
100.0 \% \\
15.2 \%\end{array}$ \\
\hline & $\begin{array}{l}\text { No } \\
\text { contesto }\end{array}$ & $\begin{array}{l}0 \\
0.0 \% \\
0.0 \%\end{array}$ & $\begin{array}{l}5 \\
41.7 \% \\
16.1 \% \\
\end{array}$ & $\begin{array}{l}1 \\
8.3 \% \\
4.2 \%\end{array}$ & $\begin{array}{l}3 \\
25.0 \% \\
12.5 \% \\
\end{array}$ & $\begin{array}{l}1 \\
8.3 \% \\
10.0 \% \\
\end{array}$ & $\begin{array}{l}2 \\
16.7 \% \\
33.3 \% \\
\end{array}$ & $\begin{array}{l}0 \\
0.0 \% \\
0.0 \%\end{array}$ & $\begin{array}{l}12 \\
100.0 \% \\
12.1 \%\end{array}$ \\
\hline & $\begin{array}{l}\text { No } \\
\text { consume }\end{array}$ & $\begin{array}{l}0 \\
0.0 \% \\
0.0 \%\end{array}$ & $\begin{array}{l}1 \\
33.3 \% \\
3.2 \% \\
\end{array}$ & $\begin{array}{l}1 \\
33.3 \% \\
4.2 \% \\
\end{array}$ & $\begin{array}{l}0 \\
0.0 \% \\
0.0 \%\end{array}$ & $\begin{array}{l}0 \\
0.0 \% \\
0.0 \%\end{array}$ & $\begin{array}{l}0 \\
0.0 \% \\
0.0 \%\end{array}$ & $\begin{array}{l}1 \\
33.3 \% \\
100.0 \%\end{array}$ & $\begin{array}{l}3 \\
100.0 \% \\
3.0 \% \\
\end{array}$ \\
\hline \multicolumn{2}{|l|}{ Total } & $\begin{array}{l}3 \\
3.0 \% \\
100.0 \%\end{array}$ & $\begin{array}{l}31 \\
31.3 \% \\
100.0 \%\end{array}$ & $\begin{array}{l}24 \\
24.2 \% \\
100.0 \%\end{array}$ & \begin{tabular}{|l|}
24 \\
$24.2 \%$ \\
$100.0 \%$
\end{tabular} & $\begin{array}{l}10 \\
10.1 \% \\
100.0 \%\end{array}$ & $\begin{array}{l}6 \\
6.1 \% \\
100.0 \%\end{array}$ & $\begin{array}{l}1 \\
1.0 \% \\
100.0 \%\end{array}$ & $\begin{array}{l}99 \\
100.0 \% \\
100.0 \%\end{array}$ \\
\hline
\end{tabular}

Fuente: Obtenido del análisis de datos de la muestra.

El 54.2\% del segmento meta tiene un ingreso entre $\$ 8000$ y $\$ 12000$ al mes.

La mayor parte que paga ese precio y esta dispuesto a consumirlo tiene un ingreso familiar de más de $\$ 8000$ por mes.

La información estableció que el consumo de huevo libre de jaula es de una clase media baja a media alta.

$\mathrm{H}_{0}=$ La variable consumo es independiente de la variable integrantes que forman su familia.

$\mathrm{H}_{\mathrm{a}}=\mathrm{La}$ variable consumo es dependiente de la variable integrantes que forman su familia.

Tabla 10. Pruebas de chi-cuadrado entre la variable consumo y número de integrantes de la familia.

\begin{tabular}{|l|l|l|l|}
\hline & Valor & Gl & Sig. asintótica (2 caras) \\
\hline Chi-cuadrado de Pearson & $25.555^{\mathrm{a}}$ & 3 & .000 \\
Razón de verosimilitud & 17.352 & 3 & .001 \\
Asociación lineal por lineal & 16.105 & 1 & .000 \\
N de casos válidos & 101 & & \\
\hline
\end{tabular}

Fuente: Obtenido del análisis de datos de la muestra. $\alpha=0.05$, gl $=3$. Valor crítico $=7.8147$. Valor calculado $=25.555$ 
Con el valor crítico obtenido que es menor que el valor calculado se rechaza la hipótesis nula ya que el resultado de correlación entre las variables muestra la dependencia entre la variable de consumo con la variable número de integrantes de la familia.

Tabla 11. Relación entre consumo y número de integrantes que forman su familia.

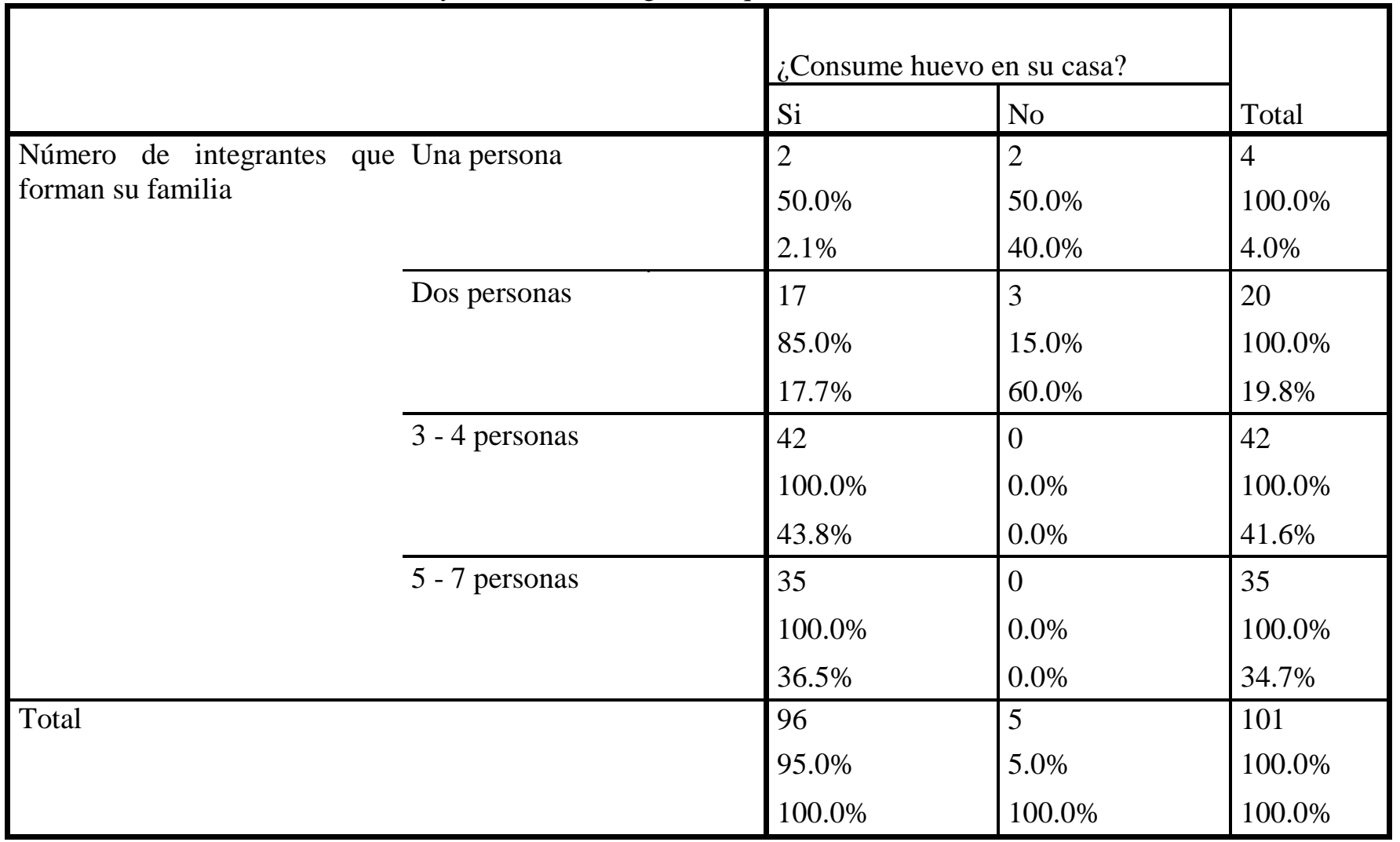

Fuente: Obtenido del análisis de datos de la muestra.

Como se observa en la tabla anterior el $80.5 \%$ de los respondientes que si consumen huevo sus familias están integradas por más de 3 personas, siendo así que el $43.8 \%$ lo conforman de 3 a 4 personas y el restante de 5 a 7 integrantes por familia.

$\mathrm{H}_{0}=\mathrm{La}$ variable precio que paga actualmente es independiente de la variable integrantes que forman su familia.

$\mathrm{H}_{\mathrm{a}}=\mathrm{La}$ variable precio que paga actualmente es dependiente de la variable integrantes que forman su familia.

Tabla 12. Pruebas de chi-cuadrado entre las variables precio y número de integrantes que forman su familia.

\begin{tabular}{|l|l|l|l|}
\hline & Valor & Gl & Sig. asintótica (2 caras) \\
\hline Chi-cuadrado de Pearson & $48.473^{\mathrm{a}}$ & 18 & .000 \\
Razón de verosimilitud & 44.076 & 18 & .001 \\
Asociación lineal por lineal & 15.763 & 1 & .000 \\
N de casos válidos & 100 & & \\
\hline
\end{tabular}

Fuente: Obtenido del análisis de datos de la muestra. $\alpha=0.05, \mathrm{gl}=18$. Valor crítico $=28.8693$. Valor calculado $=48.473$ 
Con el valor crítico obtenido que es menor que el valor calculado se rechaza la hipótesis nula ya que el resultado de correlación entre las variables muestra la dependencia entre la variable precio que paga actualmente con la variable número de integrantes de la familia

Tabla 13. Relación entre precio que paga actualmente y número de integrantes de su familia.

\begin{tabular}{|c|c|c|c|c|c|c|c|c|c|}
\hline & \multicolumn{7}{|c|}{ Precio que paga actualmente } & \multirow[b]{2}{*}{ Total } \\
\hline & & $\begin{array}{l}\$ 25-\$ \\
30\end{array}$ & $\begin{array}{l}\$ 31 \\
\$ 35\end{array}$ & $\begin{array}{l}\$ 36 \\
\$ 40\end{array}$ & $\begin{array}{l}\$ 41 \\
\$ 45\end{array}$ & $\begin{array}{l}\$ 46 \\
\$ 50\end{array}$ & $\begin{array}{|ll|}\text { Más de } \\
\text { \$51 }\end{array}$ & $\begin{array}{l}\text { No } \\
\text { consume }\end{array}$ & \\
\hline \multirow[t]{4}{*}{$\begin{array}{l}\text { Número de } \\
\text { integrantes que } \\
\text { forman su familia }\end{array}$} & $\begin{array}{l}\text { Una } \\
\text { persona }\end{array}$ & $\begin{array}{l} \\
50.0 \% \\
10.0 \% \\
\end{array}$ & $\begin{array}{l}0 \\
0.0 \% \\
0.0 \% \\
\end{array}$ & $\begin{array}{l}0 \\
0.0 \% \\
0.0 \% \\
\end{array}$ & $\begin{array}{l}0 \\
0.0 \% \\
0.0 \% \\
\end{array}$ & $\begin{array}{l}0 \\
0.0 \% \\
0.0 \%\end{array}$ & $\begin{array}{l}0 \\
0.0 \% \\
0.0 \% \\
\end{array}$ & $\begin{array}{l}2 \\
50.0 \% \\
40.0 \% \\
\end{array}$ & $\begin{array}{l}4 \\
100.0 \% \\
4.0 \% \\
\end{array}$ \\
\hline & $\begin{array}{l}\text { Dos } \\
\text { personas }\end{array}$ & $\begin{array}{l}3 \\
15.0 \% \\
15.0 \% \\
\end{array}$ & $\begin{array}{l}2 \\
10.0 \% \\
7.4 \% \\
\end{array}$ & $\begin{array}{l}7 \\
35.0 \% \\
26.9 \% \\
\end{array}$ & $\begin{array}{l}1 \\
5.0 \% \\
16.7 \% \\
\end{array}$ & $\begin{array}{l}2 \\
10.0 \% \\
66.7 \% \\
\end{array}$ & $\begin{array}{l}2 \\
10.0 \% \\
15.4 \% \\
\end{array}$ & $\begin{array}{l}3 \\
15.0 \% \\
60.0 \%\end{array}$ & $\begin{array}{l}20 \\
100.0 \% \\
20.0 \%\end{array}$ \\
\hline & $\begin{array}{l}3 \quad-4 \\
\text { personas }\end{array}$ & $\begin{array}{l}7 \\
17.1 \% \\
35.0 \% \\
\end{array}$ & $\begin{array}{l}11 \\
26.8 \% \\
40.7 \% \\
\end{array}$ & $\begin{array}{l}16 \\
39.0 \% \\
61.5 \% \\
\end{array}$ & $\begin{array}{l}1 \\
2.4 \% \\
16.7 \% \\
\end{array}$ & $\begin{array}{l}0 \\
0.0 \% \\
0.0 \%\end{array}$ & $\begin{array}{l}6 \\
14.6 \% \\
46.2 \% \\
\end{array}$ & $\begin{array}{l}0 \\
0.0 \% \\
0.0 \%\end{array}$ & $\begin{array}{l}41 \\
100.0 \% \\
41.0 \%\end{array}$ \\
\hline & $\begin{array}{l}5-7 \\
\text { personas }\end{array}$ & $\begin{array}{l}8 \\
22.9 \% \\
40.0 \% \\
\end{array}$ & \begin{tabular}{|l|}
14 \\
$40.0 \%$ \\
$51.9 \%$ \\
\end{tabular} & $\begin{array}{l}3 \\
8.6 \% \\
11.5 \% \\
\end{array}$ & $\begin{array}{l}4 \\
11.4 \% \\
66.7 \% \\
\end{array}$ & $\begin{array}{l}1 \\
2.9 \% \\
33.3 \% \\
\end{array}$ & $\begin{array}{l}5 \\
14.3 \% \\
38.5 \% \\
\end{array}$ & $\begin{array}{l}0 \\
0.0 \% \\
0.0 \%\end{array}$ & $\begin{array}{l}35 \\
100.0 \% \\
35.0 \%\end{array}$ \\
\hline Total & & $\begin{array}{l}20 \\
20.0 \% \\
100.0 \%\end{array}$ & $\begin{array}{l}27 \\
27.0 \% \\
100.0 \%\end{array}$ & $\begin{array}{l}6 \\
26.0 \% \\
100.0 \% \\
\end{array}$ & $\begin{array}{l}6 \\
6.0 \% \\
100.0 \% \\
\end{array}$ & \begin{tabular}{|l|}
3 \\
$3.0 \%$ \\
$100.0 \%$ \\
\end{tabular} & $\begin{array}{l}13 \\
13.0 \% \\
100.0 \%\end{array}$ & $\begin{array}{l}5 \\
5.0 \% \\
100.0 \%\end{array}$ & $\begin{array}{l}100 \\
100.0 \% \\
100.0 \% \\
\end{array}$ \\
\hline
\end{tabular}

Fuente: Obtenido del análisis de datos de la muestra.

Tal como se muestra en la tabla, el segmento meta el $46.2 \%$ está conformado de 3-4 personas por familia y el $38.5 \%$ lo forman de 5-7 integrantes por familia.

Teniendo el dato del número de familias que conforman nuestro mercado objetivo 6852, el
13.7\% y sabiendo que 5797 familias en cada compra adquieren un kilogramo de huevo y el resto $2 \mathrm{kgs}$. Se cruza información con la tabla 14 para determinar en números el mercado potencial de kilogramos existente en la ciudad, obteniendo lo siguiente:

Tabla 14. Distribución del mercado objetivo por frecuencia y cantidad de consumo.

\begin{tabular}{|c|c|c|c|}
\hline & 1 kg. & 2 kgs. & Total kgs. anuales \\
\hline Cada 2 días & $\begin{array}{c}348 \text { familias } \\
\text { (63 474 kgs. anuales) }\end{array}$ & 0 & 6344 \\
\hline 1 vez por semana & $\begin{array}{c}\text { 3 } 912 \text { familias (172 } 118 \mathrm{kgs} . \\
\text { anuales) }\end{array}$ & $\begin{array}{c}\text { 904 familias (47 024 } \\
\text { kgs. anuales) }\end{array}$ & 219142 \\
\hline Cada 15 días & $\begin{array}{c}2124 \text { familias } \\
\text { (46 722 kgs. anuales) }\end{array}$ & $\begin{array}{c}151 \text { familias (3 923 kgs. } \\
\text { anuales) }\end{array}$ & 59645 \\
\hline 1 vez por mes & 348 familias (4 173 kgs. anuales) & 0 & 4173 \\
\hline Total & 5797 familias & 1055 familias & $\mathbf{3 3 7 4 3 4 ~ k g s}$ \\
\hline
\end{tabular}

Fuente: Obtenido del análisis de datos de la muestra. 
Definiendo con precisión el mercado objetivo al cual el huevo libre de jaula tiene que ser dirigido en la región de Delicias, se tiene una importante ventaja con respecto a la creación de las líneas estratégicas de comercialización, ya que al conocer los consumidores se puede satisfacer la necesidad de consumo que presentan.

\section{CONCLUSIONES}

Después de analizar e interpretar la información obtenida respecto al mercado de huevo libre de jaula o libre pastoreo se concluye que respecto a la demanda existente del consumo de huevo en la ciudad se identifica que es un producto de primera necesidad ya que el $95 \%$ de la población lo adquiere, mínimo una vez por semana la cantidad de un kilogramo, sin importar el nivel de estudios, sexo y edad. Siendo de su preferencia el huevo blanco y que el tamaño, limpieza y precio son los criterios tomados en cuenta para la adquisición del producto. Además de que prefieren como lugar de compra el supermercado, seguido por las tiendas de abarrotes y fruterías; y el precio que pagan actualmente por kilogramo oscila entre los \$ 36 y \$ 40. Por otro lado, la oferta determinada que existe en la ciudad es de aproximadamente 20000 piezas por semana, mismas que son surtidas diariamente en los locales establecidos y reconocidos por CANACO.

La distribución que se deberá de seguir por parte de la empresa debe de ser diaria o al menos cada tercer día en establecimientos comerciales localizados en los sectores anteriormente mencionados, en presentación de 24 piezas o en bolsas. $\mathrm{Y}$ eficientar la producción del huevo para lograr ofrecerlo a un precio entre los $\$ 40$ y $\$ 60$ para de esta manera mantener su punto de equilibrio y lograr mejores utilidades y mayor rentabilidad con las ventas.

Determinando el mercado objetivo que será nuestro consumidor potencial de huevo de libre pastoreo lo constituye el $13.7 \%$ de los núcleos familiares existentes en Delicias o dicho en otras palabras 6852 familias mismas que se ubican en su mayoría en los sectores oriente y poniente de la ciudad de Delicias. De las cuales 5797 familias lo compran en presentaciones de $1 \mathrm{~kg}$. Y el resto en presentaciones de $2 \mathrm{~kg}$. Elevando estas cantidades con la frecuencia que adquieren al año, se concluye que el mercado potencial que demanda este producto en Delicias es de 337434 kgs. anuales, adicionando con lo que actualmente se distribuye en Chihuahua y la producción con la que cuente la empresa se concluye que se cubre perfectamente esta demanda.

\section{LITERATURA CITADA}

Avila Rivera, S. C. (1 de Diciembre de 2015).

Plan rector del sistema producto

orgánico del Estado de Chihuahua.

Delicias , Chihuahua, Mexico: PIFI 2014-2015.

Borbón Riveros , S. L., Chaves Chinome, L. M., \& Moreno Cabra, D. M. (2017). Plan de Negocios Huevo Organico. Bogotá: Universidad Santo Tomás. https://doi.org/10.15332/tg.pre.2020. 00128

FAO. (2018). FAO. Obtenido de FAO: http://www.fao.org/docrep/007/ad818s/a d818s03.htm\#TopOfPage

Garcia Hernández, S. (05 de Julio de 2018). Avicultura. Obtenido de Avicultura: https://www.avicultura.mx

George, D., \& Mallery, P. (2003). SPSS for Windows step by step: A simple guide 
and reference, 11.0 update. Obtenido de wps. ablongman.

com/wps/media/objects/385. George

4answers pdf.

Gómez, M. A., Gómez, L., \& Schwentesius, R. (2002). Dinamica del mercado internacional de productos orgánicos y las perspectivas para México. Momento Económico, 54-68.

Kotler, P., \& Armstrong, G. (2008).

Fundamentos de marketing. Pearson.

Management. (11 de Octubre de 2013). Huevo orgánico, un mercado muy nutritivo.

Dinero en Imagen, pág. 1.
Reyes Hernández, U. (6 de Diciembre de 2014). Emprendices. Obtenido de emprendices: https://www.emprendices.co/plan-denegocios-para-una-granja-productorade-huevo-organico/

Thovar Bermejo , V. (2019). Imsextremadura.educarex.es. Obtenido de lmsextremadura.educarex.es: https://lmsextremadura.educarex.es/plug infile.php/28239/mod_resource/content/ 1/huevos1.pdf

Valadez Delgado, R. (2018). Avicultura mx. De nuevo México en 2018 rompera record como país con mayor consumo percapita de huevo en el mundo.

Copyright (c) 2019 José E đuardo Magaña Magaña, Jacqueline López Rodriguez, Christian Mauricio Kiessling Davi son, Laura Escalera Ochoa, Julio César Barrera Torres

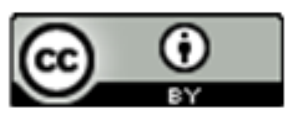

Este texto está protegido por una licencia licencia CreativeCommons 4.0.

Usted es libre para Compartir —copiar y redistribuir el $\mathrm{m}$ aterial en cualquier medio o formato- $\mathrm{y}$ Adaptar el documento —remezclar, transformar y crear a partir del material- para cualquier propósito, incluso para fines comerciales, siempre que cumpla la condición de:

Atribución: Usted debe dar crédito a la obra original de manera adecuada, proporcionar un enlace a la licencia, e in dicar si se han realizado cambios. Puede hacerlo en cualquier forma razonable, pero no de forma tal que sugiera que tiene el apoyo del licenciante o 10 recibe por el uso que hace de la obra.

Resumendelicencia - Textocompletodelalicencia 